\title{
Preclinical and clinical issues in Alzheimer's disease drug research and development
}

\author{
Cesare Mancuso $^{1 *}$ and Silvana Gaetani ${ }^{2}$ \\ ${ }^{1}$ Institute of Pharmacology, Catholic University School of Medicine, Rome, Italy \\ 2 Department of Physiology and Pharmacology "Vittorio Erspamer," Sapienza University of Rome, Rome, Italy \\ *Correspondence: cmancuso@rm.unicatt.it
}

Edited and reviewed by:

Salvatore Salomone, Catania University, Italy

Keywords: Alzheimer's disease, preclinical studies, clinical trials as topic, drug research and development, neurodegeneration

Alzheimer's disease (AD) is a chronic, rapidly progressive neurodegenerative disease, characterized by loss of memory and cognitive abilities, inability to carry out regular daily activities and mental disorders. The following numbers provide an idea of the extent of the problem: 36 million people worldwide suffer from dementia, of which 7.7 million new cases each year, and $60-70 \%$ of them are diagnosed with AD. Patients with AD deal with numerous problems, one of the most relevant being the lack of drugs that can slow down or hinder disease onset. Indeed, the currently available drugs, namely acetylcholinesterase inhibitors donepezil, rivastigmine, and galantamine, and the NMDA receptor antagonist, memantine, are able to counteract or delay the symptoms related to cognitive decline and nearly entirely in individuals with mild-to-moderate $\mathrm{AD}$. The shortage of drugs available for the treatment of $\mathrm{AD}$ is also related to the difficulties faced by many pharmaceutical companies, including some Big Pharma, that despite significant achievements from their hit/leads in preclinical and early stages of clinical research, have not been able in recent years, to launch their products on the market due to ineffective results in terms of efficacy or safety of advanced clinical trials. This problem is at least in part due to investigators' difficulties in identifying the most adequate outcomes in order to plan clinical trials. Indeed, in many cases, attention has been focused on certain plasma or cerebral-spinal fluid (CSF) biomarkers, both for the relative ease of sampling and for the possibility they could offer to monitor the disease progression. However, it is worth emphasizing, that despite clinical trials based on the use of peripheral biomarkers have provided encouraging results on the ability of some drugs to block the production or deposition of $\beta$-amyloid $(A ß)$ or even destroy existing senile plaques, key phase III studies, specifically designed to evaluate the improvement of cognitive activity, have shown a reduced ability of these new molecules to enhance memory or the ability to perform regular daily living functions in patients with $\mathrm{AD}$ or, in some cases have also resulted in severe side effects. Another limitation of pharmacological research on $\mathrm{AD}$ is the scarce ability to enhance collaboration between pharmaceutical industries and public or private non-profit organizations conducting basic research, such as universities. Both these sectors would benefit from the strengthening of this cooperation allowing non-profit institutions to enhance basic research on $\mathrm{AD}$ by receiving funds from pharmaceutical companies that, in turn, could benefit from the research of qualified scientists worldwide.

This Research Topic has been designed as a platform to analyze some of the most relevant problems posed by research on $\mathrm{AD}$, suggest and develop new ideas that may improve both preclinical and clinical research on this disease. This Research Topic in which leading experts in the field of $\mathrm{AD}$ have contributed, is organized in 5 review articles, 3 original research articles, 1 opinion article, 1 Perspective article and 1 Commentary.

The complexity of the events that determine the onset and progression of $\mathrm{AD}$ is emphasized by the many factors that contribute to the pathogenesis of this disease. Recently, what has been pointed out is the pathogenetic role played by posttranslational modifications, such SUMOylation, on proteins involved in the production of Aß (Nisticò et al., 2014). In light of this, it is worth noting the remarkable discovery in $\mathrm{Tg} 2576$ transgenic mice, a validated preclinical model for the study of $\mathrm{AD}$, that the imbalance of the phenomena of protein SUMOylation/deSUMOylation occurs in cognitive brain areas, such as the hippocampus and cortex, in the very early stages of the disease. This finding certainly contributes to indicate an alternative target for the development of new drugs for $\mathrm{AD}$ (Nisticò et al., 2014). The importance of having predictive preclinical models for the proper study of $\mathrm{AD}$ is also pointed out by Davis and Head (2014) who suggest the aged canine as an useful animal species for studies on $\mathrm{AD}$ and as an alternative to rodent models. Indeed, it has been shown that elderly beagle dogs have a progressive loss of cognitive functions with age and they show characteristic neuropathological hallmarks of AD (Davis and Head, 2014). This canine model allowed preclinical studies on the role of neuroprotective drugs, such as statins. An interesting link between the pathogenesis of $\mathrm{AD}$ and of other, apparently different, diseases, such as glaucoma, has been highlighted in a study by Trovato Salinaro et al. (2014). In fact, both AD and glaucoma have a common pathogenetic role played by free radicals and the ability to activate the adaptive stress response systemically (Trovato Salinaro et al., 2014). As in many cells, the activation of cell stress response is a response mechanism to the damage caused by free radicals and its modulation by nutritional supplements, defined nutraceuticals, is currently considered an adjuvant approach to standard therapy of diseases, including AD (Mecocci et al., 2014). The results by Scuderi et al. (2014) seem to support this theory, showing that 
the up-regulation of the sirtuin system, via resveratrol, is able to reduce the activation of astrocytes and the production of inflammatory mediators in primary cultures of rat astrocytes treated with $A ß(1-42)$. The causal role of infectious agents in relation to the pathogenesis of $\mathrm{AD}$ has been little experimented. As reported by Piacentini et al. (2014), recurrent infection by herpes simplex virus type I (HSV-1) can be considered a risk factor for $A D$. The mechanism(s) through which HSV-1 could give rise to AD are not completely understood, even if the HSV-1-mediated proteolytic processing of APP, generating also $A \beta 40$ and $A \beta 42$ monomers or small oligomers, is among those which received the greatest consensus (Piacentini et al., 2014).

Among the issues with the highest translational impact on research in the field of $\mathrm{AD}$ is the use of "old" drugs such as vitamin D, currently used for years in the treatment of bone loss, above all in the elderly, whose neuroprotective properties in subjects with $\mathrm{AD}$ have been recently brought to light (Annweiler et al., 2014). A very updated and comprehensive review article by Cornelius et al. (2014) examines the factors that can be considered as a common denominator of $\mathrm{AD}$ and osteoporosis. The dysregulation of the endocannabinoid system, initially based on a number of preclinical evidence, as a viable mechanism to intervene in the pathogenesis of $\mathrm{AD}$ is no longer controversial (Aso and Ferrer, 2014). Furthermore, the availability of "new" pharmacological tools to modulate the endocannabinoid system of the brain, one with the preclinical evidence of their effectiveness in terms of a significant reduction of oxidative and metabolic damage load on neurons and objective improvements of behavior in individuals with $\mathrm{AD}$, leads research further toward the study of the endocannabinoid system as a novel therapeutic target in AD (Aso and Ferrer, 2014). An example of how a virtuous collaboration between non-profit institutions, such as universities, and pharmaceutical companies should develop is in the article by Lundkvist et al. (2014). This paper focuses on the activities of "AlzeCure," a non-profit Swedish foundation founded by brilliant researchers with a strong background in R\&D from a Big Pharma such as Astra Zeneca. The AlzeCure acts as a catalyst, receiving continuous feedback from biotech companies, and leading academic institutions (e.g., Karolinska Institute) involved in $\mathrm{R} \& \mathrm{D}$ of drugs for $\mathrm{AD}$, collaborating in the preclinical and clinical field, demonstrating that a useful collaboration between non-profit institutions and pharmaceutical companies is actually possible (Lundkvist et al., 2014). A viable alternative to this model of academic-industry cooperation is the one proposed by Beauchet et al. (2014) who, in their Commentary, described the activities of "Biomathics," an emerging scientific research consortium whose task is to promote cooperation among the academic research teams working in the field of aging and longevity. One of the main goals of this Consortium is to foster the creation of large research groups, formed by scientists worldwide, so as to increase the possibility of applying and obtaining research grants to enhance international basic research (Beauchet et al., 2014).

\section{ACKNOWLEDGMENTS}

As guest-editors of this research topic, we warmly thank all the authors of the contributions that have allowed us to give rise to an issue of great scientific interest and relevance. We also thank the devoted reviewers who have provided the authors with effective and useful suggestions for the very high quality improvement of the contributions. One last non-ritual appreciation is addressed to all the members of the Editorial Office of Frontiers in Pharmacology who have certainly contributed together with authors and reviewers in making this research topic a real success with their patient help.

\section{REFERENCES}

Annweiler, C., Karras, S. N., Anagnostis, P., and Beauchet, O. (2014). Vitamin D supplements: a novel therapeutic approach for Alzheimer patients. Front. Pharmacol. 5:6. doi: 10.3389/fphar.2r.2014.00006

Aso, E., and Ferrer, I. (2014). Cannabinoids for treatment of Alzheimer's disease: moving toward the clinic. Front. Pharmacol. 5:37. doi: 10.3389/fphar.2r.2014.00037

Beauchet, O., Merjagnan, C., and Annweiler, C. (2014). From industrial research to academic discoveries, towards a new concept of partnership: the Biomathics model. Front. Pharmacol. 5:166. doi: 10.3389/fphar.2r.2014. 00166

Cornelius, C., Koverech, G., Crupi, R., Di Paola, R., Angela, K., Lodato, F., et al. (2014). Osteoporosis and Alzheimer pathology: role of cellular stress response and hormetic redox signaling in aging and bone remodeling. Front. Pharmacol. 5:120. doi: 10.3389/fphar.2r.2014.00120

Davis, P. R., and Head, E. (2014). Prevention approaches in a preclinical canine model of Alzheimer's disease: benefits and challenges. Front. Pharmacol. 5:47. 10.3389/fphar.2014.00047

Lundkvist, J., Halldin, M. M., Sandin, J., Nordvall, G., Forsell, P., Svensson, S., et al. (2014). The battle of Alzheimer's Disease - -the beginning of the future Unleashing the potential of academic discoveries. Front. Pharmacol. 5:102. doi: 10.3389/fphar.2014.00102

Mecocci, P., Tinarelli, C., Schulz, R. J., and Polidori, M. C. (2014). Nutraceuticals in cognitive impairment and Alzheimer's disease. Front. Pharmacol. 5:147. doi: 10.3389/fphar.2014.00147

Nisticò, R., Ferraina, C., Marconi, V., Blandini, F., Negri, L., Egebjerg, J., et al. (2014). Age-related changes of protein SUMOylation balance in the A $\beta P P$ Tg2576 mouse model of Alzheimer's disease. Front. Pharmacol. 5:63. doi: 10.3389/fphar.2014.00063

Piacentini, R., De Chiara, G., Li Puma, D. D., Ripoli, C., Marcocci, M. E., Garaci, E., et al. (2014). HSV-1 and Alzheimer's disease: more than a hypothesis. Front. Pharmacol. 5:97. doi: 10.3389/fphar.2014.00097

Scuderi, C., Stecca, C., Bronzuoli, M. R., Rotili, D., Valente, S., Mai, A., et al. (2014). Sirtuin modulators control reactive gliosis in an in vitro model of Alzheimer's disease. Front. Pharmacol. 5:89. doi: 10.3389/fphar.2014. 00089

Trovato Salinaro, A., Cornelius, C., Koverech, G., Koverech, A., Scuto, M., Lodato, F., et al. (2014). Cellular stress response, redox status, and vitagenes in glaucoma: a systemic oxidant disorder linked to Alzheimer's disease. Front. Pharmacol. 5:129. doi: 10.3389/fphar.2r.2014.00129

Conflict of Interest Statement: The authors declare that the research was conducted in the absence of any commercial or financial relationships that could be construed as a potential conflict of interest.

Received: 29 September 2014; accepted: 07 October 2014; published online: 28 October 2014.

Citation: Mancuso $C$ and Gaetani S (2014) Preclinical and clinical issues in Alzheimer's disease drug research and development. Front. Pharmacol. 5:234. doi: 10.3389/fphar.2014.00234

This article was submitted to Experimental Pharmacology and Drug Discovery, a section of the journal Frontiers in Pharmacology.

Copyright (c) 2014 Mancuso and Gaetani. This is an open-access article distributed under the terms of the Creative Commons Attribution License (CC BY). The use, distribution or reproduction in other forums is permitted, provided the original author(s) or licensor are credited and that the original publication in this journal is cited, in accordance with accepted academic practice. No use, distribution or reproduction is permitted which does not comply with these terms. 